Recepción: 28 / 03 / 2017

Aceptación: 13 / 05 / 2017

Publicación: 15 / 07 / 2017

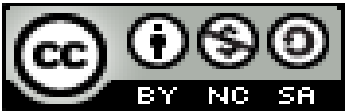

Ciencias técnicas y aplicadas

Artículo de investigación

\title{
La importancia de la topografía en las ingenierías y arquitectura
}

\section{The importance of topography in engineering and architecture}

\section{A importância da topografia em engenharia e arquitetura}

Julio C. Castro-Moreira ${ }^{\mathrm{I}}$

julio.castrom@gmail.com

Martha A. Vélez-Gilces II martha.velezg@hotmail.com

Correspondencia: julio.castrom@gmail.com

\footnotetext{
I Magister en Gerencia Educativa, Ingeniero Civil, Docente de la Universidad Laica Eloy Alfaro de Manabí, Manta, Ecuador.

${ }^{\text {II }}$ Magister en Gerencia Educativa, Ingeniero Civil, Universidad Laica Eloy Alfaro de Manabí, Manta, Ecuador.
} 


\section{Resumen}

La topografía, como disciplina que se encarga de describir de una forma muy detallada la superficie de un terreno, es beneficiosa para muchos ámbitos de la vida. Este trabajo presenta como objetivo exponer la importancia de la topografía en las ingenierías y la arquitectura. Para ello se realiza un análisis de fuentes primarias y secundarias, a través de los diferentes motores de búsqueda de internet. Se incluye una explicación de lo que se entiende por topografía y algunos elementos relacionados con ella, y se muestra la importancia del uso de la misma en las diferentes ingenierías.

Palabras clave: Topografía; ingeniería agrícola; ingeniería mecánica; ingeniería industrial; ingeniería eléctrica; ingeniería civil; arquitectura.

\section{Abstract}

Topography, as a discipline that is responsible for describing in a very detailed way the surface of a terrain, is beneficial to many areas of life. This paper aims to expose the importance of topography in engineering and architecture. For this, an analysis of primary and secondary sources is carried out, through the different internet search engines. It includes an explanation of what is meant by topography and some elements related to it, and shows the importance of using it in different engineering.

Keywords: Topography; agricultural engineering; mechanical engineering; industrial engineer; electric engineering; civil engineering; architecture..

\section{Resumo}

A topografia, como uma disciplina que é responsável por descrever de forma muito detalhada a superfície de um terreno, é benéfica para muitas áreas da vida. Este artigo pretende expor a importância da topografia em engenharia e arquitetura. Para isso, é realizada uma análise de fontes primárias e secundárias, através dos diferentes mecanismos de busca na internet. Inclui uma explicação sobre o que se entende por topografia e alguns elementos relacionados a ele, e mostra a importância de usá-lo em engenharia diferente.

Palavras chave: Topografia; engenharia agrícola; engenharia mecânica; engenharia industrial; engenharia elétrica; engenharia civil; arquitetura. 


\section{Introducción}

La topografía es una de las artes más antiguas e importantes que practica el hombre, porque desde los tiempos más antiguos ha sido necesario marcar límites y terrenos. En la era moderna, la topografía se utiliza extensamente, los resultados de los levantamientos topográficos de nuestros días se emplean por ejemplo, para:

- Elaborar planos de superficies terrestres, arriba y abajo del mar.

- Trazar cartas de navegación para uso en el aire, tierra y mar.

- Establecer límites en terrenos de propiedad privada y pública.

- La topografía es esencial en varios campos; por ejemplo:

- Agrimensura

- Arqueología

- Arquitectura

- Geografía

- Ingeniería de minas

- Ingeniería Geográfica

- Ingeniería Catastral y Geodesia

- Ingeniería Forestal

- Ingeniería Agrícola

- Ingeniería Civil

- Ingeniería sanitaria

- Minería

- Sistemas de Información Geográfica

- Batimetría 
- Oceanografía

- Cartografía

- Alcantarillados Diseño de vías

- Túneles

- Ingeniería Petrolera

- Ingeniería Ambiental

- Ingeniería en Transporte y Vías de Comunicación

- Ingeniería pesquera

- Agronomía

Todos los ingenieros y arquitectos deben conocer los usos de la topografía, es por eso que este trabajo presenta como objetivo exponer la importancia de la topografía en las ingenierías y la arquitectura.

Para ello se realiza un análisis de fuentes primarias y secundarias, a través de los diferentes motores de búsqueda de internet.

\section{Desarrollo}

La topografía, es la ciencia que estudia los objetivos de la superficie de la tierra, con sus formas y detalles, tanto naturales como artificiales o ficticios. Es una disciplina o técnica que se encarga de describir de una forma muy detallada la superficie de un terreno, pero no solo se limita a realizar la elevación de campos en los terrenos sino que posee componentes edición y redacción cartográfica, que se encarga de estudiar las elaboraciones de los mapas geográficos (Conceptodefinición, s/f).

La topografía (de topos, "lugar", y grafos, "descripción"), con sus formas y detalles, tanto naturales como artificiales (ver planimetría y altimetría). Esta representación tiene lugar sobre superficies planas, limitándose a pequeñas extensiones de terreno, utilizando la denominación de geodesia para áreas mayores. (Fundación Wikimedia, 2017) 
El principal objetivo de los proyectos de ingeniería de un levantamiento topográfico es determinar la posición relativa entre varios puntos sobre un plano horizontal. Esto se realiza mediante un método llamado planimetría. El siguiente objetivo es determinar la altura entre varios puntos en relación con el plano horizontal definido anteriormente, la altimetría. Esto se lleva a cabo mediante la nivelación directa. Tras ejecutar estos dos objetivos, es posible trazar planos y mapas a partir de los resultados obtenidos consiguiendo un levantamiento tipográfico.

Por lo tanto se puede hablar de varias partes de la topografía:

a. Planimetría: Consiste en proyectar sobre un plano horizontal los elementos de la cadena o poligonal sin considerar su diferencia de elevación.

b. Altimetría: Estudia las diferencias de elevación de los puntos sobre la superficie terrestre, dando su posición relativa o absoluta, proyectado sobre un plano vertical y referida a un plano de comparación cualquiera o a una superficie de comparación como el nivel medio del mar.

c. Planialtimetría: Estudia los métodos y procedimientos de medición y representación gráfica de los elementos que componen las cadenas planimétrica y altimétrica simultáneamente.

A las mediciones y recopilaciones de datos suficiente al terreno que se desea representar, se le conoce como levantamiento topográfico.

Las mayores fuentes de error, según la literatura consultada son:

- Errores de medidas de ángulos y distancias.

- Selección deficientes de estaciones, que resultan en malas condiciones de visado debidas a:

- Sol y sombra alternadas

- Visibilidad de la parte superior del estadal solamente

- Visado hacia donde está el sol.

- No hacer el doble visado, o no duplicar los ángulos de deflexión. 
Los errores que contienen las medidas son de dos tipos:

a Sistemáticos: Se conforman en las leyes matemáticas y físicas. Su magnitud puede ser constante o variable, dependiendo de las condiciones. Los errores sistemáticos, pueden calcularse y eliminarse sus defectos, aplicando correcciones. Por ejemplo; una cinta de $30 \mathrm{~m}$ que tiene una longitud mayor en $0,005 \mathrm{~m}$, introducirá un error positivo de $0,005 \mathrm{~m}$ $(5 \mathrm{~mm})$ cada vez que se utiliza. El cambio de longitud de una cinta de acero resulta de una diferencia dada de temperaturas.

b. Accidentales: Son los que quedan después de haber eliminado las equivocaciones y los errores sistemáticos. Son ocasionados por factores que quedan fuera de control del observador, obedecen las leyes de la probabilidad. Estos errores están presentes en todas las mediciones topográficas.

La escala es el concepto fundamental en las representaciones gráficas, bien sean cartas, mapas, planos, croquis u otras gráficas. Se define como "la relación existente entre la medida gráfica del dibujo y la real del terreno".

El Teodolito es un aparato que posee múltiples usos en topografía, se usa principalmente para medir ángulos horizontales y verticales, distancias por taquimetría y para trazar alineamientos rectos.

La topografía no sólo se limita a realizar los levantamientos de campo en terreno, sino que posee componentes de edición y redacción cartográfica, para que al confeccionar un plano se pueda entender el fonema representado a través del empleo de símbolos convencionales y estándares, previamente normados para la representación de los objetos naturales y antrópicos en los mapas o cartas topográficas.

La topografía es de suma importancia para todos aquellos que desean realizar estudios de ingeniería en cualquiera de sus ramas, así como para los estudiantes de arquitectura, no solo por los conocimientos y habilidades que puedan adquirir, sino por la influencia didáctica de su estudio.

La topografía tiene gran importancia en la aplicación y proyección de diseños arquitectónicos y de ingeniería, ya que es la base en la que un diseño o futuro proyecto deberá emplazarse. 
También es muy importante para replanteos no solo de diseños a emplazarse, sino también el reelevamiento de edificaciones ya consolidadas. La topografía es una rama que no puede desligarse de carreras afines a esta como las ingenierías, agronomía, arquitectura, geodesia, y muchas más (Arquiben Consultores, s/f).

Los proyectos de ingeniería en levantamiento topográfico consisten en hacer una topografía de un lugar, es decir, llevar a cabo la descripción de un terreno en concreto. Con los datos obtenidos en un levantamiento tipográfico se pueden trazar mapas o planos en los que aparte de las características mencionadas anteriormente, también se describen las diferencias de altura de los relieves o de los elementos que se encuentran en el lugar donde se realiza el levantamiento.

Los proyectos de ingeniería de levantamientos topográficos y la topografía en general, tienen una gran importancia en el desarrollo de proyectos de construcción de infraestructuras debido a la evolución y avance que se ha producido en esta ciencia por la ayuda de las nuevas tecnologías que permiten llevar a cabo mediciones y descripciones más precisas y exactas; por eso una medida mal tomada o un plano mal realizado puede tener graves consecuencias pues eso supondría una incorrecta representación de la realidad que impediría llevar a cabo construcciones en dicho terreno.

La topografía tiene aplicaciones dentro de ingeniería agrícola:

- En levantamientos como trazos,

- Deslindes,

- Divisiones de tierra,

- Determinación de área,

- Levantamientos catastrales hechos con el propósito de localizar límites de propiedad y valorar los inmuebles.

Según Ruiz Vizcaíno (2014), la importancia de la Topografía en la Agricultura se debe a que es un valioso auxiliar en el mejoramiento, conservación y rehabilitación de los suelos. En la actividad profesional de la agricultura, los planos topográficos constituyen un elemento de trabajo esencial para el estudio de proyectos de riego y de drenaje, ya que a la hora de regar el terreno 
debe de estar nivelado. Es importante la topografía en la agricultura para la nivelación de tierras, manejo del agua, problemas de salinidad, además en la medición de superficies, trazo de canales, drenes, regaderas, surquerías, la apreciación de desniveles y pendientes; el trazado de líneas de pendientes predeterminadas y curvas de nivel; tendido de alambrados; la señalización del curso del avión en tareas de fumigación; etc. También es importante para las actividades de marcación de tajamares de aguada y la distribución de agua para el campo, la orientación y replanteo de plantaciones, instalaciones rurales y obras de jardinería, etc. (Ruiz Vizcaino, 2014).

En la actividad profesional de la agricultura, los planos topográficos constituyen un elemento de trabajo esencial para el estudio de proyectos de riego y de drenaje, ya que a la hora de regar el terreno debe de estar nivelado (Luzania, 2014).

Brevé (1998) presenta la utilidad de la topografía en la agricultura en los siguientes puntos:

- Replantear datos de un piano en el campo y viceversa.

- Localizar y marcar los límites de una propiedad.

- Medir el área de una propiedad.

- Subdividir terrenos en parcelas.

- Medir áreas en parcelas.

- Obtener información (elevaciones, distancias, curvas de nivel) para proyectos de riego, drenaje y conservación de suelos (Brevé, 1996).

La topografía tiene aplicaciones dentro de la ingeniería eléctrica:

- En los levantamientos previos y los trazos de líneas de trasmisión,

- Construcción de plantas hidroeléctricas,

- En instalación de equipos para plantas nucleoeléctricas, etc.

La topografía tiene aplicaciones dentro de la ingeniería industrial:

- Para la instalación precisa de máquinas y equipos industriales, 
- Configuración de piezas metálicas de gran precisión, etc. (Alcantara García, 2014)

La topografía tiene aplicaciones dentro de la ingeniería mecánica:

- Para el alineamiento óptico.

La topografía tiene aplicaciones dentro de la ingeniería minera:

- Para el levantamiento y trazo de túneles, galerías y lumbreras,

- Para las cuantificaciones de volúmenes extraídos, etc.

La topografía tiene aplicaciones dentro de la ingeniería geológica:

- En la relación de las formaciones geológicas,

- Determinación de configuraciones de cuencas hidrológicas,

- Como apoyo fundamental de la fotogeología, etc.

La topografía tiene aplicaciones dentro de la ingeniería civil:

En ella es necesario realizar trabajos topográficos antes, durante y después de la construcción de obras tales como carreteras, ferrocarriles, edificios, puentes, canales, presas, fraccionamientos, servicios municipales etc.

Es utilizada como un servicio para los distintos sectores de obra como son: excavadores, armadores, carpinteros, soldadores, etc.

La topografía es fundamental en la ejecución de la obra, debiéndose realizar con tres premisas fundamentales: responsabilidad, velocidad y sencillez. (Irving, 2009)

- Responsabilidad: porque la ejecución de la obra se realiza en base a las referencias que topografía marca. Una marca mal realizada representa un trabajo posterior sin sentido por no estar ubicada en el lugar que corresponde.

- Velocidad: el retraso en las marcas representa el retraso en la obra, ya que nadie puede realizar su tarea si no sabe dónde hacerla.

- Sencillez: marcas complicadas de comprender o de utilizar son motivo de errores. 
Los levantamientos preliminares son de suma importancia, ya que de ellos depende la puesta en obra del proyecto. En el caso de ser una obra de remodelación o montaje en un edificio ya construido resulta de suma importancia la obtención con precisión de la posición de columnas, muros, vigas, etc. Para la ejecución de esos relevamientos se pueden seguir diferentes métodos según las circunstancias.

Las nivelaciones forman parte fundamental también en los trabajos topográficos, siendo casi en su totalidad nivelaciones del tipo geométricas (Suárez, 2015).

En cuanto a la arquitectura, la topografía es fundamental para el correcto emplazamiento de estas en la superficie terrestre, por ejemplo los desniveles de terreno obligan a levantar información topográfica para el emplazamiento exacto y calculado de dichas construcciones, para la penetr4ación de construcciones en superficies Terrestres, para las superficies de terreno con pendientes pronunciadas, etc. (Universidad Mayor de San Simón, s/f).

Hablar de Topografía y Arquitectura, es generar una simbiosis de conocimientos, que permitan analizar y de tal manera entender el espacio físico con todos su componentes, esto es fundamental a la hora de enfrentar un proyecto de diseño, pues conceptos como tipología, plástica, enrasamiento, pliegues y otros, aunque parecieran de una sola disciplina, al final se conjugan, convergen al interpretar la información sea esta extraída de un levantamiento topográfico o de una anteproyecto arquitectónico, donde la sinuosidad de las curvas de nivel denotan estas características y permiten establecer necesidades conjuntas entre el topógrafo y el arquitecto, los cuales aportarán desde su disciplina formas de modificar y/o representar los terrenos donde se construirán los futuros proyectos. (Duque Arango, s/f)

\section{Conclusiones}

La práctica de la topografía es compleja pero es de mucha utilidad para todos los proyectos de ingeniería, tanto en la fase de su elaboración como en la de su ejecución. Es por ello que los estudiantes de ingeniería y arquitectura deben prestar atención a los contenidos sobre topografía que se imparten en cada carrera.

\section{Referencias bibliográficas}

Alcantara García, D. (2014). Topografía y sus aplicaciones México. 
Arquiben Consultores. (s/f). ¿Para qué sirve la topografía en la arquitectura?

Brevé, M. (1996). Manual de clases: principios de topografía aplicados al área agrícola.

CONCEPTODEFINICION. (s/f). Definición de Topografía. from http://conceptodefinicion.de/topografia/

DUQUE ARANGO, J. (s/f). La Topografía y la Arquitectura. from http://www.elagrimensor.com.ar/elearning/lecturas/LA\%20TOPOGRAFIA\%20Y\%20LA\%20AR QUITECTURA.pdf

$\begin{array}{llll}\text { Fundación } & \text { Wikimedia. } & \text { (2017). } & \text { Topografía. }\end{array}$ https://es.wikipedia.org/wiki/Topograf\%C3\%ADa

Irving, I. (2009). Importancia de la Topografia from http://ingcivilabarca.blogspot.com/

Luzania, E. (2014). Topografia en la Agricultura from https://prezi.com/-vqydid6vpec/topografiaen-la-agricultura/

Ruiz Vizcaino, D. C. (2014). Topografía Aplicada a la Agricultura from https://prezi.com/8h3gojx7drdx/topografia-aplicada-a-la-agricultura/

SUAREZ, W. (2015). Campo de Acción de la Topografía. from http://sancheszuares.blogspot.com/2015/07/campo-de-accion.html

Universidad Mayor de San Simón. (s/f). Topografía en la Arquitectura from https://sites.google.com/site/topografiaenlaarquitectura/ 